\title{
Commercial herbal medicines used as African traditional medicines: Ngoma Herbal Tonic Immune Booster interferes with a rapid urine drug screening test
}

\author{
M E Mothibe, ${ }^{1}$ MSc (Med) (Pharmacology), N Dip Med Tech, PhD; E Osuch, ${ }^{2}$ MD, PhD, FCP (ACCP), MSc (Med) Pharmacol, \\ Dip Fam Med; C P Kahler-Venter, ${ }^{2}$ MSc (Med) (Pharmacology), PhD \\ ${ }^{1}$ Department of Pharmaceutical Sciences, School of Pharmacy, Sefako Makgatho Health Sciences University, Pretoria, South Africa \\ ${ }^{2}$ Department of Pharmacology and Therapeutics, School of Medicine, Sefako Makgatho Health Sciences University, Pretoria, South Africa
}

Corresponding author: ME Mothibe (mamza.mothibe@smu.ac.za)

\begin{abstract}
Background. The prevalent use of African traditional medicine by the general public has been reported. With commercialisation and marketing, some of the herbal medicines (HMs) used are readily available over the counter, most of them promoted as immune boosters. These commercial HMs have not been taken through clinical trials and other tests that would validate their composition and safety, and other properties such as their effect on laboratory diagnostic tests.

Objective. To investigate the cross-reactivity of selected HMs with commonly tested drugs of abuse (DoA) using a qualitative rapid urinalysis assay.

Methods. The six HMs selected were bought from local pharmacies. A rapid urinalysis screening test was performed with the Instant View Multi-Drug of Abuse Test kit from Labstix Diagnostics. Drug-free urine (DFU) was pooled from samples donated by healthy volunteers. Urine samples that had tested positive for DoA were obtained from a pharmacology laboratory. Aliquots of the urine samples were spiked with the HMs in neat and diluted form, and tested at various time intervals.

Results. The results for the DFU samples spiked with the HMs remained negative. There were no significant changes in $\mathrm{pH}$ or specific gravity of the samples. The results of samples that had tested positive for tetrahydrocannabinol (THC) were not altered by five of the HMs when spiked at $40 \% \mathrm{v} / \mathrm{v}$. The HM Ngoma Herbal Tonic Immune Booster caused false-negative results for the THC test.

Conclusion. An important finding is that the herbal mixture Ngoma Herbal Tonic Immune Booster caused false-negative results for the cannabinoid screening test. It adds to the list of substances that may be potential adulterants of urine for screening tests.
\end{abstract}

S Afr Med J 2017;107(9):734-737. DOI:10.7196/SAMJ.2017.v107i9.12197

The prevalent use of African traditional medicine (ATM) by the general public in South Africa (SA) has been reported. ${ }^{[1]}$ It has been documented that most people consult a traditional health practitioner before a primary health practitioner, ${ }^{[2]}$ and may not disclose this fact during consultation with a healthcare provider ${ }^{[3]}$ As a result of commercialisation and marketing, some herbal medicines (HMs) are readily available over the counter, most of them being punted as immune boosters. The use of these commercial herbal medicines (CHMs) with modern packaging and marketing practices is said to be widespread in SA. ${ }^{[4]}$ The National Department of Health in SA has made progress towards institutionalisation and regulation of traditional medicine, ${ }^{[5]}$ but there are as yet no regulations that govern the labelling and packaging of CHMs used as ATM.

Since these herbal mixtures have not been tested and validated, the effect of their presence on laboratory diagnostic tests is unknown. During the 2010 FIFA World Cup hosted in SA, a concern was raised that the use of ATMs extracted from local plants may have granted unfair advantage to soccer players who used them, as they may contain undetectable amounts of stimulants. ${ }^{[6]}$ The officials were concerned that some herbal materials used may have produced compounds undetectable by the laboratory tests available at the time, while other products of the medicinal plants used may not have been included in the list of banned substances of the World Anti-Doping Agency (WADA). The only plants or plant-related compounds on the official list of WADA are cannabis, cocaine and ephedra, and they are listed as stimulants. ${ }^{[6,7]}$ There are no listed compounds related to ATM.

However, there could be more issues concerning the use of HMs by sportspeople. The HMs may have properties similar to some banned substances, so that when tested, a sportsperson could unknowingly test positive for banned substances. Common substances known to cause false-positive results on urine screening tests for drugs of abuse (DoA) include the Vicks inhaler testing positive for amphetamine, non-steroidal anti-inflammatory drugs such as ibuprofen testing positive for barbiturates, and cannabinoids and some fluoroquinolones testing positive for opiates. ${ }^{[8,9]} \mathrm{Ma}$ huang, a Chinese HM, caused a false-positive screening test for amphetamines, which was confirmed to be a cross-reaction of the ephedrine in the product. ${ }^{[10]}$

Another area of concern is adulteration of samples. Adulteration is a process of deliberate interference with the process of specimen collection, transport or analysis, with the intention of avoiding a positive test outcome. In vivo adulteration is the intake of interfering substances such as drugs, or lots of water, before sample collection. In vitro adulteration is when substances are added into the urine sample after collection to alter the results, mainly to cause a false negative. Substances commonly used for adulteration include drug-free human, animal or synthetic urine or assay interferants. ${ }^{[11,12]}$ 
Generally, urine is tested routinely for DoA in health facilities to aid the diagnosis of potential abusers and some psychiatry patients on admission. The other important testing is for banned substances in special populations such as those whose work environment requires that they be drug free, and people involved in professional sport. Qualitative screening tests are initially used to test for the presence of the DoA, as they are quick, sensitive, relatively economical and simple to use. ${ }^{[8,11]}$ Results of screening assays are used to indicate whether further testing is required (as illustrated in Fig. 1) and provide a guide for follow-up action from a clinical perspective.

A rapid urinalysis assay based on an immunoassay method is the screening test commonly used for DoA. It is based on the principle of antigen-antibody reaction in which the drug or drug metabolite present in urine will interact with a labelled polyclonal or monoclonal antibody provided. ${ }^{[13,14]}$ Various substances may interfere with immunoassays by cross-reacting with the test reagents and resulting in false-negative, false-positive or invalid results.

\section{Objective}

Literature searches provided no results for cross-reactivity of ATMs, or the influence of ATMs on laboratory tests. This study therefore sought to establish whether any of the commonly used CHMs may affect the outcomes of a rapid qualitative screening test for DoA. It was intended to show whether the presence of the CHMs would alter the results of a rapid urinalysis assay.

\section{Methods}

Permission for the study (ref. no. MREC/M/09/2011:PG) was granted by the Sefako Makgatho Health Sciences University (SMU) Research Committee and the Superintendent of Dr George Mukhari Academic Hospital.

\section{The rapid urinalysis assay}

The test kit used was the Instant View Multi-Drug of Abuse Test kit from Labstix Diagnostics (SA). It consists of test cassettes, each with six panels for concurrent testing of amphetamine, cocaine, methamphetamine, morphine, tetra-hydrocannabinol (THC) and methylenedioxymethamphetamine. The test is a one-step lateral flow chromatographic immunoassay. Each panel contains drug-protein conjugate immobilised on a porous membrane support. The test is based on the principle of competition for limited binding sites on the antibody between the drug or drug metabolite present in the urine

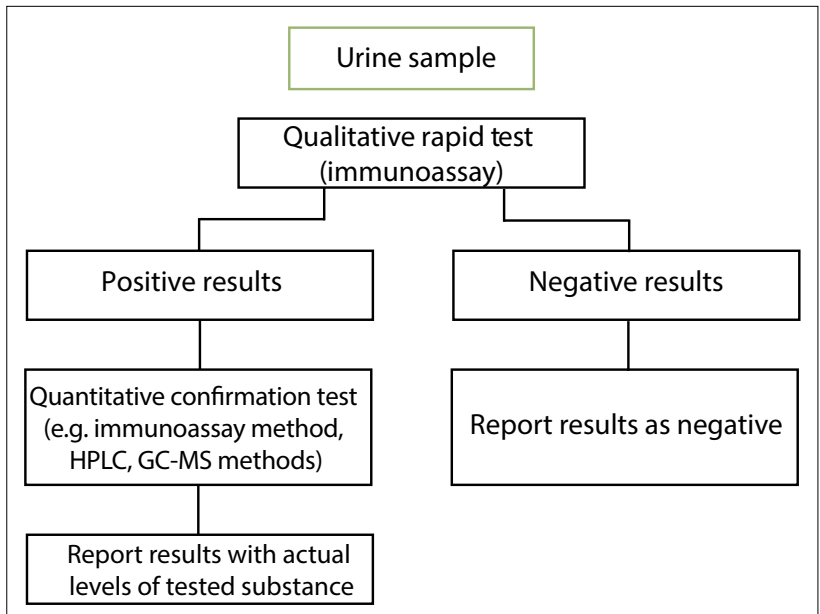

Fig. 1. Flow diagram for routine urine testing for drugs of abuse. (HPLC $=$ high-performance liquid chromatography; GC-MS = gas chromatographymass spectrometry). sample and the immobilised drug-protein conjugate. If the drug is absent in the urine, or is present in below cut-off levels, a coloured conjugate will interact with the drug antigen immobilised in the test line and form a coloured line. If the drug or metabolite is present, it will bind to and saturate all the binding sites on the antibody conjugate, preventing any binding by the drug antigen at the test area. The results are negative if two colour lines appear, one in the test area and the other in the control area, regardless of the intensity of the line. The results are positive when only one colour line appears in the control site and are invalid if no colour line appears on the test as well as the control site.

A 10-test dipstick kit from Labstix Diagnostics was used to test qualitatively for $\mathrm{pH}$ and specific gravity (SG).

\section{The herbal mixtures and preparation}

The six CHMs selected for the study are used as immune boosters or to strengthen the body and are commonly sold in many retail outlets; they were bought from local pharmacies. They were Intlamba Zifo (denoted HM1 for the purposes of this study), Maphilisa HM (HM2), Matla African Medicine for All Diseases (HM3), Ngoma Herbal Tonic Immune Booster (HM4), Stametta Body Healing Liquid (HM5) and Vuka Uphile Immune Booster (HM6). From each of the six CHMs, three serial dilutions of 10, 100 and 1000 times were made with distilled water. These HMs were taken through the test procedure outlined in Fig. 2.

\section{Testing drug-free urine (DFU)}

Ten urine samples were collected from healthy volunteers, each of whom confirmed that they had not taken any drugs, HMs or overthe-counter medicines within the preceding 7 days. The samples were pooled as DFU. Both the individual samples and the DFU were tested with the dipsticks for $\mathrm{pH}$ and SG. The remaining pooled DFU was stored in the refrigerator at $4^{\circ} \mathrm{C}$.

Four samples of $9 \mathrm{~mL}$ each of DFU were made for each of the six CHMs. A volume of $1 \mathrm{~mL}$ each of the neat $\mathrm{HM}$ and the three dilutions were added to the samples. A dipstick test was done on each spiked DFU sample and the results were recorded. The spiked DFU samples were then tested using the Instant View Multi-Drug of Abuse Test cassette, following the instructions in the test kit manual. The test was repeated at time intervals of $8,24,48,72,96$ and 120 hours and the results were recorded. Following up on the results of the test, six aliquots of the DFU were each spiked with the HMs at $40 \% \mathrm{v} / \mathrm{v}$ $(2400 \mu \mathrm{L} \mathrm{DFU}+1600 \mu \mathrm{L} \mathrm{CHM})$. The control for each sample was composed of $2400 \mu \mathrm{L} \mathrm{DFU}$ plus $1600 \mu \mathrm{L}$ of distilled water to make up for the dilution.

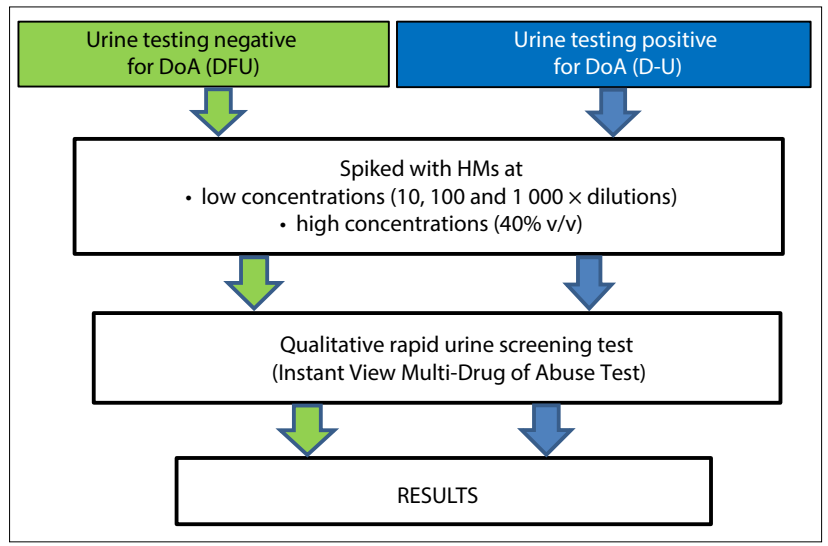

Fig. 2. Outline of the method used. (DoA = drugs of abuse; $D F U=$ drug-free urine; $D-U=$ positive urine samples; HMs $=$ herbal medicines.) 


\section{Testing urine positive for DoA}

Urine samples that had been sent to SMU's Department of Pharmacology and Therapeutics laboratory for routine testing and had tested positive for any of the tested substances were collected. The positive urine samples (D-U) were labelled accordingly, and centrifuged for 5 minutes at $1000 \mathrm{rpm}(250 \mathrm{~g})$. Each supernatant was collected and stored in the refrigerator at $4^{\circ} \mathrm{C}$ until needed.

Six aliquots of the D-U samples were each spiked with one of the six HMs at $40 \% \mathrm{v} / \mathrm{v}$. The positive control for each sample was composed of $2400 \mu \mathrm{L} \mathrm{D}-\mathrm{U}$ plus $1600 \mu \mathrm{L}$ DFU. These samples were tested following the same procedure according to the test kit instructions.

\section{Results}

\section{Testing DFU}

The urine samples obtained from healthy volunteers appeared normal. The colour of the samples was various shades of amber, but none of the observed parameters deviated from the normal ranges as indicated by the dipstick test. The $\mathrm{pH}$ and $\mathrm{SG}$ of the individual urine samples differed, but the pooled sample values were $\mathrm{pH} 6.0$ and SG 1.020 .

The results of the rapid qualitative test on the spiked DFU samples were all negative for the tested DoA and remained negative from day 1 till day 5 . The dipstick results for $\mathrm{pH}$ and $\mathrm{SG}$ also remained the same for each of the samples throughout the 5 days of testing.

The results for the $40 \% \mathrm{v} / \mathrm{v}$ spiked urine samples were also negative, and remained negative throughout the 5 days. The mean SG and $\mathrm{pH}$ for the DFU spiked with the HMs were 1.020 and 5.5, respectively.

\section{Testing urine that was positive for DoA}

Eight urine samples (D-U) that had tested positive for THC were used in this assay. The $\mathrm{pH}$ of samples ranged from 5 to 7.5 and the SG from 1.005 to 1.030 . The $\mathrm{pH}$ of the controls was between 5 and 6.5 and the SG between 1.010 and 1.030. The controls for all the $\mathrm{D}-\mathrm{U}$ samples remained positive for THC. The results of seven D-U samples spiked with HM1, HM2, HM3, HM5 and HM6 remained positive for THC in all concentrations of the HMs tested, from the lowest to the highest $(40 \% \mathrm{v} / \mathrm{v})$.

The D-U samples spiked with HM4 at the highest concentration $(40 \% \mathrm{v} / \mathrm{v})$ tested negative for THC. Because this HM was the only one that was alcohol based, alcohol had to be excluded as a possible cause of the false-negative result. A $13.5 \%$ methanol solution was used to spike some D-U samples, which were then taken through the same procedure. The results of the spiked D-U samples remained positive, confirming that alcohol did not alter the results.

\section{Discussion}

Five of the six HMs tested had no influence on the quantitative rapid urinalysis assay. HM4, Ngoma Herbal Tonic Immune Booster, showed false-negative results for THC in the urine samples that were positive for THC when spiked at $40 \% \mathrm{v} / \mathrm{v}$ with HM4. It is stated on the container of this HM that it contains Sutherlandia, Echinacea, dandelion, alfalfa (lucerne), Aloe ferox, Harpagophytum (devil's claw) and alcohol (13.5\%). Alcohol was excluded as a possible cause of the false-negative result. The plausible explanation for the false-negative result could therefore be that alcohol as a solvent extracted some lipophilic compounds from the plants used, which would not be extracted by water as a solvent. These compounds would have then interfered with the test reagents, resulting in the false-negative results.

For the test to be positive, the drug metabolites in the urine sample compete for binding on the immobilised protein conjugate (antibody) with the colour-coded drug antigen on the test panel. A negative test result occurs when the colour-coded drug antigen binds to the antibody. The compounds in HM4 interacted with the test reagents and the THC in the sample in such a way that the drug metabolite could not bind to the antibody.

There are several ways in which false-negative results could occur for a particular drug when using immunoassays. A false negative may occur when the drug is present in the sample but the detection limit of the method is too high, or when the actual concentration of the drug in the sample is very low. ${ }^{[8,13]}$ Most screening tests have cut-off concentrations above which the results would be reported as positive. ${ }^{[8,14-16]}$ It may also be caused by cross-reactivity of the antibody in the assay, the time lapse between drug ingestion and specimen collection, or adulteration of the sample. ${ }^{[15]}$

For HM4, the false-negative results occurred at high concentration $(40 \% \mathrm{v} / \mathrm{v})$. Although the test used is a qualitative test, it has a quantitative part due to the cut-off levels, which give a guide to the possible concentration of the substance if positive. ${ }^{[14]}$ The suggestion here is that the presence of HM4 at low levels, which are the most likely in vivo physiological levels, would not affect the screening test. The high concentration used would be most likely to be achieved by in vitro sample manipulation, therefore suggesting that HM4 could be an adulterant for THC. Dilution of a sample, which would normally cause reduced concentration to below cut-off levels ${ }^{[1]}$ is not a factor in this case because the control sample remained positive.

The false-negative results could also have been caused by crossreactivity of the antibody in the assay with one or more plant compounds present in the HM. As is well known in HMs, there could be undeclared ingredients in the mixture owing to either deliberate or unintentional contamination, ${ }^{[4,17]}$ and also because manufacturers of commercial HMs protect their recipes, as they consider themselves to hold unofficial patents thereof. ${ }^{[18]}$ Screening tests are designed to detect classes of drugs, so although they are sensitive they have low specificity. ${ }^{[1]}$ THC is a highly lipid-soluble compound of plant origin. It could be that the antibody to THC in the assay has variable degrees of reactivity or affinity towards drugs or metabolites with identical chemical structure to THC, or with unrelated chemical structures. HM4 may contain one or more plant compounds that are structurally related to THC, since THC is of plant origin. Plant compounds that are extracted in alcohol include glycosides, sugars, amino acids, terpenoids, alkaloids and polyphenols. ${ }^{[1,20]}$ Whether these were the compounds that interfered with the test is speculative at this stage, as there could be more compounds present. The result cannot be extrapolated to physiological levels, as it was observed at a high concentration ( $40 \% \mathrm{v} / \mathrm{v})$ of HM4. However, the result permits the possibility of the risk of the use of HM4 as an adulterant. The cannabinoid assay was recognised to be the assay most susceptible to false-negative results after six out of 16 chemicals commonly used as adulterants reported false-negative results. ${ }^{[21]}$ The substances interfering with cannabinoid immunoassays included ibuprofen, tolmetin ${ }^{[10]}$ and common household chemicals such as drain cleaner, ammonia and bleach. ${ }^{[15,21]}$ This is the first report of an ATM, particularly one available over the counter, being a potential adulterant to a cannabinoid urine screening test using a quantitative lateral flow chromatography immunoassay. The outcome also signifies the importance of appropriate labelling of CHMs, which may provide information on the range of possible compounds that may be present in a herbal mixture.

\section{Study limitations}

The sample set for this study was small, so a wider study with more samples and replicates and including more herbal medicinal products 
should be done. The study method used a rapid screening test from only one manufacturer. The results may therefore not be generalisable to the test kits from other manufacturers.

\section{Conclusion}

Of the six HMs tested, only one showed the potential of interfering with a rapid, qualitative urine test for DoA. Ngoma Herbal Tonic Immune Booster caused false-negative results for THC. This is the first finding of the possibility of commercial HMs used as ATMs interfering with rapid, qualitative screening tests. The findings therefore facilitate the route for further studies investigating whether the use of these medicines interferes with diagnostic tests in general.

Acknowledgements. We thank the SMU Department of Pharmacology and Therapeutics laboratory staff members, who assisted with acquiring the urine samples.

Author contributions. MEM conducted the research as part of her PhD studies, and drafted the manuscript. EO and CPKV were co-supervisor and supervisor, respectively; they proofread the draft. All authors approved the final manuscript.

Funding. Sefako Makgatho Health Sciences University.

Conflicts of interest. None

1. Peltzer K. Utilisation and practice of traditional/complementary/alternative medicine (TM/CAM) in South Africa. Afr J Trad Complement Altern Med 2009;6(2):175-185. https://www.ncbi.nlm.nih.gov/ pmc/articles/PMC2816568/ (accessed 6 August 2017).

2. Truter I. African traditional healers: Cultural and religious beliefs intertwined in a holistic way S Afr Pharm J 2007;74(8):56-60. https://www.sapj.co.za/index.php/SAPJ/article/download/239/231 (accessed 6 August 2017).

3. Ernst E. Prevalence of use of complementary medicine: A systematic review. Bull World Health Organ 2000;78(2):252-257. https://doi.org/10.1590/S0042-96862000000200015
4. Ndhlala AR, van Staden J. Smokescreens and mirrors in safety and quality of herbal medicines: A case of commercialized herbal preparations. S Afr J Bot 2012;82(Sept):4-10. https://doi.org/10.1016/j. sajb. 2012.06 .008

5. South Africa. Traditional Health Practitioners Act 22 of 2007

6. Stafford L. Herbalgram: Concerns over use of African herbs during 2010 World Cup. 2010. http://cms. herbalgram.org/herbalgram/issue86/article3520.html?:issue $=86$ (accessed 8 August 2017).

7. World Anti-Doping Code. The 2015 Prohibited List. https://www.usada.org/wp-content/uploads/ wada-2015-prohibited-list-en.pdf (accessed 8 August 2017).

8. Vincent CE, Zebelman A, Goodwin C. What common substances can cause false positives on urine screens for drugs of abuse? J Fam Pract 2006;55(10):893-897. http://www.mdedge.com/sites/default/ files/issues/articles/5510JFP_ClinicalInquiries1.pdf (accessed 6 August 2017).

9. Reisfield GM, Goldberger BA, Bertholf RL. 'False-positive' and 'false-negative' test results in clinical urine drug testing. Bioanalysis 2009; (5):937-952. https://doi.org/10.4155\%2Fbio.09.81

10. Haller CA, Benowitz NL. Adverse cardiovascular and central nervous system events associated with dietary supplements containing ephedra alkaloids. N Engl J Med 2000;343:1833-1838. https://doi.org 1 10.1056\%2Fnejm200012213432502

11. Reisfield GM, Salazar E, Bertholf RL. Review: Rational use and interpretation of urine drug testing in chronic opioid therapy. Ann Clin Lab Sci 2007;37(4):301-314. http://www.annclinlabsci.org/ in chronic opioid therapy. Ann Clin Lab Sci 20
content/37/4/301.long (accessed 6 August 2017).

12. Jaffe WB, Trucco E, Levy S, Weiss RD. Is this urine really negative? A systematic review of tampering methods in urine drug screening and testing. J Subst Abuse Treat 2007;33(1):33-42. https://doi. org/10.1016/j.jsat.2006.11.008

13. Kapur BM. Drug testing methods and clinical interpretations of test results. Encyclopedia of Drugs, Alcohol and Addictive Behaviour 2001. http://www.encyclopedia.com/doc/1G2-3403100184.html (accessed 8 December 2014).

14. Moeller KE, Lee KC, Kissack JC. Urine drug screening: Practical guide for clinicians. Mayo Clin Proc 2008;83(1):66-76. https://doi.org/10.4065\%2F83.1.66

15. Uebel RA, Wium CA. Toxicological screening for drugs of abuse in samples adulterated with household chemicals. S Afr Med J 2002;92(7):547-549

16. Dasgupta A. Review of abnormal laboratory test results and toxic effects due to the use of herbal medicines. Am J Clin Pathol 2003;120(1):127-137. https://doi.org/10.1309\%2Fp024-k7vr-ddpj-ctvn

17. Kamboj A. Analytical evaluation of herbal drugs. In: Vallisuta O, Olimat SM, eds. Drug Discovery Kamboj A. Analytical evaluation of herbal drugs. In: Vallisuta O, Olimat S
Research in Pharmacognosy. InTech, 2012. https://doi.org/10.5772\%2F 26109

18. Ndhlala AR, Stafford GI, Finnie JF. et al. In vitro pharmacological effects of manufactured herbal . Ndhlala AR, Stafford GI, Finnie JF. et al. In vitro pharmacological effects of manufactured herbal
concoctions used in KwaZulu-Natal South Africa. J Ethnopharmacol 2009;122(1):117-122. https://doi. concoctions used in KwaZulu-Na
org/10.1016\%2F.j.jep.2008.12.017

19. Tiwari P, Kumar B, Kaur M, et al. Phytochemical screening and extraction: A review. Internationale Pharmaceutica Scientia 2011;1(1):98-106. https://www.scribd.com/doc/94036813/PhytochemicalScreening-and-Extraction-a-Review (accessed 6 August 2017).

20. Houghton PJ, Raman A. Laboratory Handbook for the Fractionation of Natural Extracts. London: Chapman \& Hall, 1998:113-138. https://doi.org/10.1007\%2F978-1-4615-5809-5

21. Schwarzhoff R, Cody JT. The effects of adulterating agents on FPIA analysis of urine for drugs of abuse. J Anal Toxicol 1993;17(1):14-17. https://doi.org/10.1093/jat/17.1.14

Accepted 19 April 2017 